\title{
ADVANCED CONTROL POLITICS AND OPTIMAL PERFORMANCE FOR AN IRRIGATION CANAL
}

\author{
X. Litrico* ${ }^{*}$ V. Fromion ${ }^{\dagger}$ \\ ${ }^{*}$ Cemagref, UR Irrigation, B.P. 5095, 34033 Montpellier Cedex 1, France. e-mail: xavier. litrico@cemagref.fr \\ $\dagger$ INRA, LASB, 2 place Viala, 34060 Montpellier, France. e-mail: fromion@ensam. inra. fr
}

\begin{abstract}
The paper firstly presents the model of an irrigation canal, obtained by linearizing Saint-Venant equations around a steady state. Classical control politics for an irrigation canal are then interpreted using automatic control tools. The time-delay from upstream discharge to downstream water level induces a limitation in the real-time performance of the controlled system for the so-called distant downstream control, that is not present in the local upstream control. A mixed controller combining distant downstream and local upstream control is proposed. The problem is casted into the $H_{\infty}$ optimization framework, and experimentally tested on an experimental canal located in Portugal.
\end{abstract}

Keywords: Open-channel system, Saint-Venant model, $H_{\infty}$ control, Time Delay Systems, multivariable control.

\section{Introduction}

Water demand for irrigation purposes is an increasingly important issue worldwide. Irrigation canals managers face conflicting demands: they must satisfy water users (clients that pay water and ask for an efficient service) and at the same time manage the water resource by withdrawing from the resource only the quantity of water needed.

This is a difficult task for open-channel systems, since there is a long time-delay between the upstream water resource and the user. Indeed, a drastic way to satisfy water demands would be to let the maximum discharge flow in the canal, so that user can withdraw water whenever they need it, letting the rest of the discharge flow downstream. In practise, this solution leads to a wastage of water resource, and necessitates an adaptation. However, in traditional systems, there is no global information available on the system, therefore only local regulations are possible. This has lead to the so-called water turns, where each user takes water during a pre-specified period of time. The management constraints are then entirely put on the users.

The problem considered in this paper is the following: Can automatic control techniques maintain the water distribution efficiency and at the same time improve the water resource management?

Modernization of irrigation canals, i.e. the use of automatic control techniques can modify their traditional management by using a distant downstream control [9]: in this case, the upstream gate of a pool is used to control the downstream wa- ter level of the pool. This solution leads to a economic management of the water resource, since only the necessary water is withdrawn from the resource. However, the real-time efficiency of this solution is limited by the time-delay.

We propose in the paper to use automatic control techniques to 1) interpret the two classical control structures (local upstream and distant downstream) as monovariable controllers, 2) show that the compromise between water resource management and real-time efficiency can be solved by using a multivariable controller and 3) propose a methodology to realize the desired compromise using $H_{\infty}$ optimization.

The paper is structured as follows: the model (based on SaintVenant equations) is first presented, then the two classical control structures are interpreted as particular cases of a multivariable control architecture, then a $H_{\infty}$ control method is proposed. The methodology is validated on an experimental canal located in Portugal.

\section{Canal description}

The automatic canal used in the present study is a component of the experimental facility of the Hydraulics and Canal Control Center (NuHCC) of the University of Évora (Portugal). A more detailed description is given in [7].

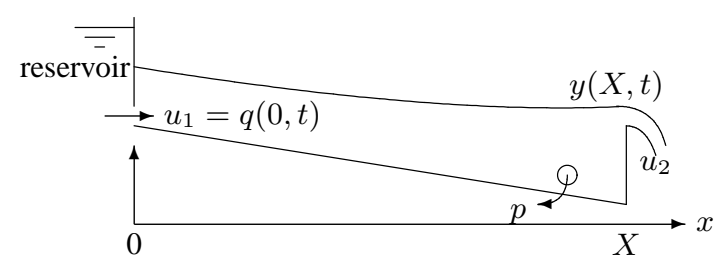

Figure 1: Schematic representation of the experimental canal

The experimental canal is a trapezoidal and lined canal, with a general cross section of bottom width $0.15 \mathrm{~m}$, sides slope 1:0.15 (V:H) and depth $0.90 \mathrm{~m}$. The last downstream $7 \mathrm{~m}$ of canal also have a rectangular cross section of width $0.7 \mathrm{~m}$. The overall canal is $145.5 \mathrm{~m}$ long and the average longitudinal bottom slope is about $1.5 \times 10^{-3}$. The design flow is $0.09 \mathrm{~m}^{3} \mathrm{~s}^{-1}$.

A longitudinal view of the canal is schematized in figure 1.

The canal inlet is equipped with a motorized flow control valve, that delivers a discharge $u_{1}$. The downstream end is controlled 
with a rectangular sluice gate $u_{2}$ (overshot gate).

An offtake $p$ is located at the downstream end of the pool, equipped with an electromagnetic flowmeter and a motorized butterfly valve.

A water level sensor is installed within an offline stilling well at the downstream end of the pool, measuring the downstream water depth $y$. The sensor is of float and counter-weight type attached by a stainless steel tape; this tape runs over a sprocket wheel. The wheel movements are transmitted to a potentiometer that transmits to the controller the analogical inputs correspondent to the water surface.

\section{Modelling of an irrigation canal}

The linearized Saint-Venant equations and the model for hydraulic structures are presented in this section, leading to a transfer matrix representation of the system (see [7] for a more complete description).

\subsection{Linearized Saint-Venant equations}

The hydraulic behavior of an open-channel irrigation canal is well described by the so-called Saint-Venant equations, which are hyperbolic nonlinear partial differential equations involving the average discharge $Q(x, t)$ and the water depth $Y(x, t)$ along one space dimension $x$ [1].

Considering small variations of water depth $y(x, t)$ and discharge $q(x, t)$ around stationary values defined by $Y_{0}(x)(\mathrm{m})$ and $Q_{0}(x)\left(\mathrm{m}^{3} / \mathrm{s}\right)$ leads to the linearized Saint-Venant equations:

$$
\begin{aligned}
L_{0} \frac{\partial y}{\partial t}+\frac{\partial q}{\partial x} & =0 \\
\frac{\partial q}{\partial t}+2 V_{0} \frac{\partial q}{\partial x}-\beta_{0} q+\left(C_{0}^{2}-V_{0}^{2}\right) L_{0} \frac{\partial y}{\partial x}-\gamma_{0} y & =0
\end{aligned}
$$

$L_{0}$ is the top width for the equilibrium regime $(\mathrm{m}), V_{0}$ the average velocity $(\mathrm{m} / \mathrm{s})$ and $C_{0}=\sqrt{\frac{g A_{0}}{L_{0}}}$ the wave celerity $(\mathrm{m} / \mathrm{s})$, where $A_{0}(x)$ is the wetted area $\left(\mathrm{m}^{2}\right)$ and $g$ the gravitational acceleration $\left(\mathrm{m} / \mathrm{s}^{2}\right)$. Moreover, one has $\gamma_{0}=$ $V_{0}^{2} \frac{\partial L_{0}}{\partial x}+g L_{0}\left[(1+\kappa) I-\left(1+\kappa-F_{0}^{2}(\kappa-2)\right) \frac{\partial Y_{0}}{\partial x}\right], \beta_{0}=$ $-\frac{2 g}{V_{0}}\left(I-\frac{\partial Y_{0}}{\partial x}\right)$ and $\kappa=\frac{7}{3}-\frac{4 A_{0}}{3 L_{0} P_{0}} \frac{\partial P_{0}}{\partial Y}$, with $S_{f 0}(x)$ the friction slope, $I$ the bed slope, $F_{0}$ is the Froude number $F_{0}=\frac{V_{0}}{C_{0}}$ and $P_{0}$ is the wetted perimeter $(\mathrm{m})$. Throughout the paper the flow is assumed to be subcritical, i.e. $F_{0}<1$.

The boundary conditions are the upstream and downstream discharges $u_{1}(t)=q(0, t)$ and $q(X, t)$, with $X$ the pool length.

The friction slope $S_{f 0}$ is modelled with Manning-Strickler formula [1]:

$$
S_{f 0}(x)=\frac{Q_{0}^{2} n^{2}}{A_{0}(x)^{2} R_{0}(x)^{4 / 3}}
$$

with $n$ the roughness coefficient $\left(\mathrm{sm}^{-1 / 3}\right)$ and $R_{0}(x)$ the hydraulic radius (m), defined by $R_{0}=A_{0} / P_{0}$.

\subsection{Linearized hydraulic structures equations}

The hydraulic structure (over shot gate) is modelled using the linearized equations [4]:

$$
q(X, t)=k_{1} y(X, t)+k_{2} u_{2}(t)
$$

with $q(X, t)$ the discharge through the structure, $y(X, t)$ the water depth upstream of the structure, $u_{2}(t)$ the sill elevation. Coefficients $k_{1}, k_{2}$ are obtained by linearizing the structure equation around a given functioning point, corresponding to the hydraulic stationary regime.

\subsection{Linear model for design}

We proposed in [5] a method to compute the transfer matrix for the linearized Saint-Venant equations. This model is aggregated with the linearized hydraulic structures equations in order to get the model for control design.

In the following, $y$ denotes the controlled water level (downstream of the pool), $G_{1}(s)$ and $G_{2}(s)$ respectively the transfer functions from $u_{1}$ and $u_{2}$ to $y$, and $\tilde{G}(s)$ the transfer function from the perturbation $p$ to $y$. The canal pool is therefore represented by:

$$
y=G_{1}(s) u_{1}+G_{2}(s) u_{2}+\tilde{G}(s) p
$$

where $y$ is the downstream water level, $u_{1}$ the upstream control (the upstream discharge $q(0, t)), u_{2}$ the downstream control (the downstream sill elevation), and $p$ is the downstream perturbation (corresponding to the unknown withdrawal). $p$ acts as an additive perturbation on the downstream discharge $q(X, t)$, therefore the transfer function is given by $\tilde{G}(s)=G_{2}(s) / k_{2}$.

\subsection{Model analysis}

We have shown in [5] that the transfer functions obtained from Saint-Venant model have the following inner-outer factorization [2]:

$$
\begin{aligned}
G_{1}(s) & =G_{1 o}(s) e^{-\tau_{1} s} \\
G_{2}(s) & =G_{2 o}(s)
\end{aligned}
$$

with $\tau_{1}$ the time-delay for downstream propagation and where $G_{1 o}$ and $G_{2 o}$ are outer.

In the general case, the delay $\tau_{1}$ is obtained as:

$$
\tau_{1}=\int_{0}^{X} \frac{d x}{V_{0}(x)+C_{0}(x)}
$$

The local transfer $G_{2}$ (relating the downstream control $u_{2}$ to the output $y$ ) is outer, while the distant one $G_{1}$ (relating the upstream control $u_{1}$ to the output $y$ ) has an inner part which is a pure time-delay. This remark may seem obvious for hydraulic engineers, but has important implications in terms of control, as will be demonstrated below. 
Remark 1 (Simplified model) In order to clarify the dynamics of the system, a simplified interpretation of this complex model is given here. In a first approximation, a canal pool can be viewed as a delayed integrator. This is in fact a good approximation of the system for low frequencies, where the mass transport is predominant. For higher frequencies, the gravity waves are predominant, one then gets an oscillating behavior.

The integrator gain can be linked to the derivative of the volume of the pool with respect to the downstream water elevation $A_{d}=\frac{\partial V}{\partial y_{X}}$. The time delay is obtained by equation (5). Including the interaction with the hydraulic structure, a low frequency approximation is therefore:

$$
G_{1}(s) \approx \frac{e^{-\tau_{1} s}}{A_{d} s+k_{1}} \quad \text { and } \quad G_{2}(s) \approx-\frac{k_{2}}{A_{d} s+k_{1}}
$$

\section{Real-time performance vs. water resource management}

As already mentioned in introduction, the control architecture for a canal pool corresponds to a given compromise between water resource management and the service to water users. In this section, it is shown that a multivariable control architecture offers enough free parameters to realize this compromise.

To this end, the two classical architectures used in canal control (local upstream and distant downstream control) are shown to be particular cases of a multivariable controller, each one being specifically in charge of one of the control objectives. This analysis finally leads to consider the multivariable architecture as a means to mix the advantages of each solution.

\subsection{Control objectives}

A naive analysis of the control problem for irrigation canals would be to consider that the control specifications can be reduced to rejecting unmeasured perturbations by controlling the downstream water level. To reduce the problem to this classical control problem neglects an essential aspect of the specifications: the water resource management. Indeed, even if a linear description of the flow is locally licit, it hides the fact that water flows from upstream to downstream. Then, the model is valid for variations around a functioning point, and the sign of the control variables corresponds to the sign of discharge variations upstream or downstream. The control architectures can now be interpreted based on this remark.

In fact, a control architecture using the upstream control variable leads to an economic water management, since e.g. faced to a decreasing demand, the upstream discharge is decreased. In other terms, the upstream control variable adapts to the consumed discharge in the pool, therefore uses only the necessary water to satisfy the effective water demand.

On the opposite, a control architecture using the downstream control variable leads to an expensive water management. Indeed, faced to a decreasing demand, the only way to maintain the downstream water level by manipulating the downstream control variable is to let the superfluous discharge go down- stream.

After this short discussion, the control objectives can be clarified:

- maintain the downstream water level $y$ by rejecting unmeasured perturbations induced by water users,

- ensure that the effect of perturbations $p$ on the downstream discharge have a zero mean value.

\subsection{Multivariable architecture: a mixed control politic}

In a canal pool, one has two control action variables $u_{1}$ and $u_{2}$ in order to control one controlled variable $y$, the downstream water level. The controller design problem leads to finding a controller $K(s)$ that relates the tracking error $e$ to the control vector $\left(u_{1}, u_{2}\right)$

$$
K(s)=\left(\begin{array}{c}
K_{1}(s) \\
K_{2}(s)
\end{array}\right)
$$

The open-loop transfer matrix is given by:

$$
G(s) K(s)=\left(G_{1}(s) K_{1}(s)+G_{2}(s) K_{2}(s)\right)
$$

As shown in this section, this controller corresponds to the combination of the two classical control architectures used in canal control. Indeed, this controller can be written as the sum of a distant downstream controller and a local upstream one:

$$
G(s) K(s)=\underbrace{G_{1}(s) K_{1}(s)}_{\text {Distant downstream contr. }}+\underbrace{G_{2}(s) K_{2}(s)}_{\text {Local upstream contr. }}
$$

\subsection{Distant downstream control of a canal}

\section{Description}

Distant downstream regulation of a canal pool consists in controlling the downstream water level $y$ by using the upstream control variable $u_{1}$. This corresponds to $K_{2}=0$, and the closed-loop system is then given by:

$$
\left\{\begin{aligned}
e & =r-y \\
u_{1} & =K_{1}(s) e \\
y & =G_{1}(s) u_{1}+\tilde{G}(s) p
\end{aligned}\right.
$$

with $r$ the reference water level and $e$ the tracking error.

The water user sees the real-time performance as the ability of the controller to reject unmeasured perturbations acting in the pool. It is characterized by the modulus of the following transfer function

$$
e=\frac{\tilde{G}(s)}{I+G_{1}(s) K_{1}(s)} p=\tilde{G}(s) S_{1}(s) p
$$

where $S_{1}$ is the sensitivity function $S_{1}=\left(1+G_{1} K_{1}\right)^{-1}$. The design consists in finding $K_{1}(s)$ such that $\left|S_{1}(j \omega)\right|$ is minimum on the largest frequency bandwidth. 


\section{Performance limitation}

We recall here an implication of a time-delay in the model. Let us define the real-time performance of the controlled system as the highest frequency $\omega_{s}$ such as $S_{1}$ stays below 1, or:

$$
\omega_{s}=\max \left\{\omega_{1}:\left|S_{1}(j \omega)\right|<1, \forall \omega<\omega_{1}\right\}
$$

Following the line of [8] and [3], it can be shown that

$$
\omega_{s} \leq \frac{\pi}{3 \tau_{1}} \approx \frac{1}{\tau_{1}}
$$

A pure time-delay therefore limits the maximal performance of a controlled system.

\subsection{Local upstream control of a canal}

\section{Description}

Local upstream regulation of a canal pool consists in controlling the downstream water level $y$ by using the downstream control variable $u_{2}$. In this case, $K_{1}=0$ and the closed-loop system is then given by:

$$
\left\{\begin{aligned}
e & =r-y \\
u_{2} & =K_{2}(s) e \\
y & =G_{2}(s) u_{2}+\tilde{G}(s) p
\end{aligned}\right.
$$

In that case, the sensitivity is given by

$$
e=\frac{\tilde{G}(s)}{I+G_{2}(s) K_{2}(s)} p=\tilde{G}(s) S_{2}(s) p
$$

The design consists in finding $K_{2}(s)$ such that $\left|S_{2}(j \omega)\right| \approx 0$ on the largest bandwidth.

\section{Performance}

Contrarily to the distant downstream case, there is no delay in the transfer function $G_{2}(s)$ (and no right half-plane zeros). Then, the achievable bandwidth has no structural limitation (even if it is in practise limited by the actuators, transmission delays and high frequency dynamics). This explains why the local upstream control is more performing than distant downstream control from a monovariable point of view (in terms of rejecting downstream perturbations).

\subsection{Combining both politics}

We have interpreted the two classical control politics for irrigation canals from a control point of view as particular monovariable cases of a multivariable controller architecture. The design specifications can now be casted into a control architecture. If the required performance can be satisfied by a distant downstream controller, then there is no need to mix the control methods. However, if the distant downstream controller cannot satisfy the specifications, it is possible to use the local upstream control for this purpose. It is then necessary to add a constraint on $u_{2}$. Since the discharge needs to come from upstream, we would like to use $u_{2}$ only for transitions, and ensure that in steady state, only $u_{1}$ has an effect on $y$.

This is a problem of actuators substitution, which can be taken into account in a cascade framework [10].

The (rapid) upstream control $u_{2}$ is used to control the output $y$ :

$$
u_{2}=K_{2}(s)(r-y)
$$

And the (slow) distant downstream control $u_{1}$ is used to regulate $u_{2}$ to a reference $r_{u_{2}}\left(r_{u_{2}}=0\right.$ in steady state).

$$
u_{1}=K_{1 b}(s)\left(r_{u_{2}}-u_{2}\right)
$$

The controller architecture can be schematized as in figure 2 .

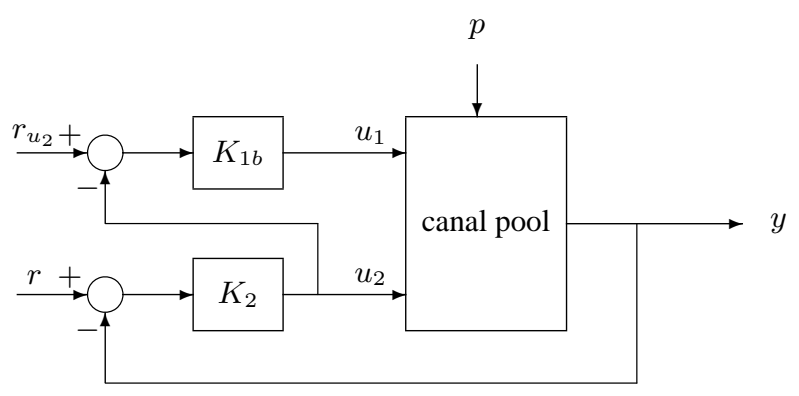

Figure 2: Cascade architecture with two control variables $\left(u_{1}, u_{2}\right)$ to control one output $y$

If $r_{u_{2}}=0$, one obtains a multivariable controller as noted above with $K_{1}=-K_{1 b} K_{2}$.

This architecture could be used to manually design simple controllers (typically PI), but it is difficult to specify robustness margins in this case.

A similar architecture is kept in the following, but recasting the problem as an $H_{\infty}$ optimization problem, which can easily take into account robustness issues. This architecture greatly simplifies the $H_{\infty}$ design presented in [6].

\section{$5 \quad H_{\infty}$ controller design}

We now propose a multivariable design method using $H_{\infty}$ optimization, in order to give a solution to the compromise between performance and resource management.

The design specifications are casted into the $H_{\infty}$ framework.

\subsection{Expression of design specifications as $H_{\infty}$ constraints}

Following the approach used in [6], design specifications are formulated using a $H_{\infty}$ 4-blocks type criteria. As a matter of fact, let us consider that the system is described by:

$$
y=G u+\widetilde{G} p
$$


The closed-loop system which links the reference, $r$, and the perturbation, $p$ to the tracking error, $e$ and the controlled input, $u$ is given by

$$
\left[\begin{array}{l}
e \\
u
\end{array}\right]=\left(\begin{array}{cc}
S & S \widetilde{G} \\
K S & K S \widetilde{G}
\end{array}\right)\left[\begin{array}{l}
r \\
p
\end{array}\right]
$$

where $S=(I+G K)^{-1}$ (sensitivity function).

The design specifications are then formulated using the following criteria, where the goal is to find the smallest $\gamma>0$ and the stabilizing controller $K$ such that

$$
\|M\|_{\infty} \leq \gamma
$$

with $M$ the augmented system as described in figure 3 .

$W_{1}, W_{1}^{-1} \in \mathcal{R} \mathcal{H}_{\infty}$ is used to specify tracking performances, perturbation rejection and modulus margin. Here, $W_{1}$ is chosen diagonal $W_{1}=\operatorname{diag}\left(W_{11}, W_{12}\right) . W_{2}, W_{2}^{-1} \in \mathcal{R} \mathcal{H}_{\infty}$ is used to specify high frequencies constraints on the controlled inputs. $W_{2}$ is also chosen diagonal $W_{2}=\operatorname{diag}\left(W_{21}, W_{22}\right)$, allowing to constrain command effort and effects of sensor noise command. $W_{3}$ is a scaling factor acting on the perturbation.

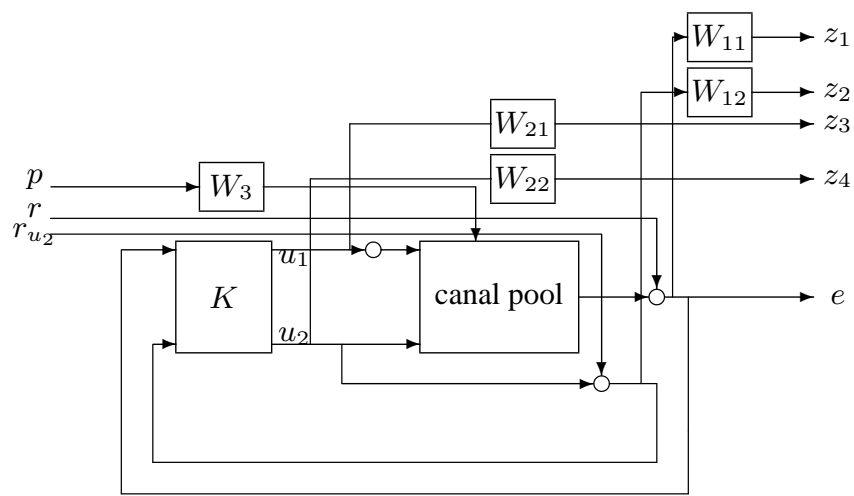

Figure 3: Augmented system for $H_{\infty}$ optimization

In order to constrain the downstream control $u_{2}$ to go asymptotically to zero, $r_{u_{2}}-u_{2}$ is feed back into the controller as a tracking error. For usual functioning, $r_{u_{2}}$ is zero, which means that specifying a weighting function $W_{12}$ with a high gain in low frequencies imposes a low value for $u_{2}$ at these frequencies.

The weighting functions $W_{i j}$ are chosen of the first order, and tuned sequentially: first $W_{22}$ is tuned to specify the maximum bandwidth for local upstream control, then $W_{11}$ is tuned to specify the global performance, then $W_{12}$ is used to specify the actuators substitution. $W_{21}$ and the scaling $W_{3}$ are used to specify control effort and robustness requirements.

The optimization resulted in $\gamma=1.1$, with a real multivariable margin (static gain margin) $\Delta G$ verifying:

$$
-8 d B \leq \Delta G \leq 15 d B
$$

and a complex margin (modulus margin) $\Delta M$ verifying:

$$
-6.4 d B \leq \Delta M \leq 6.5 d B
$$

Figure 4 presents the transfer functions with associated constraints.
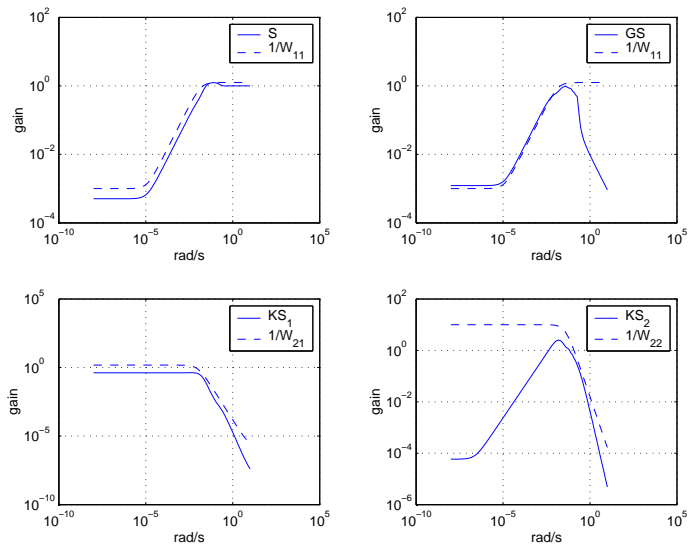

Figure 4: Closed loop transfer functions and associated frequency weighting functions

\section{$5.2 \quad H_{\infty}$ controller simulation}

Figure 5 shows how the actuators substitution works for a downstream perturbation of $0.01 \mathrm{~m}^{3} / \mathrm{s}$ : first the rapid local upstream control $u_{2}$ reacts in order to compensate for the decreasing water level, then the slow distant downstream control $u_{1}$ increases and the substitution takes place. The control $u_{2}$ goes back to zero in steady state, and the discharge is effectively delivered by $u_{1}$. The overall performance is similar to the one obtained with a pure local upstream controller.
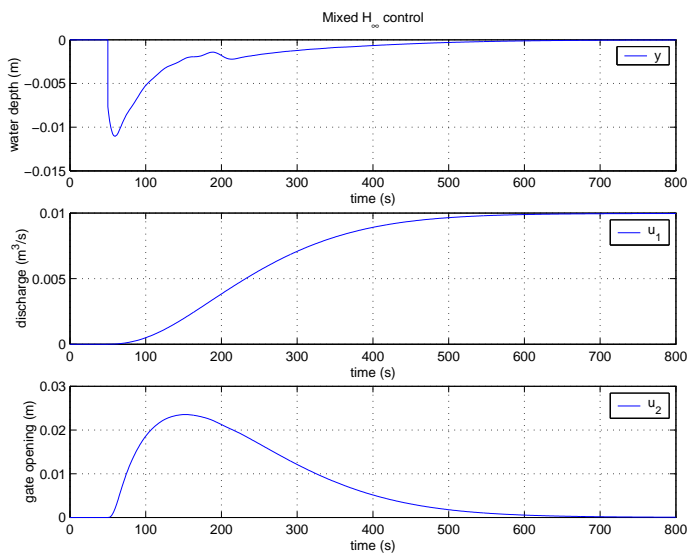

Figure 5: Actuator substitution in response to a downstream withdrawal

\subsection{Experimental results}

Figure 6 gives the experimental results obtained with the multivariable $H_{\infty}$ controller. A downstream withdrawal of $10 \mathrm{l} / \mathrm{s}$ 
$\left(0.01 \mathrm{~m}^{3} / \mathrm{s}\right)$ is done at time $t=20 \mathrm{~s}$ and stopped at time $t=950$ $\mathrm{s}$. The controller reacts as expected: first the downstream overshot gate is closed in order to maintain the output $y$ at the target $y_{c}=0.6 \mathrm{~m}$, then occurs the substitution with the upstream control; the downstream gate opens gradually while the upstream discharge increases in order to compensate for the withdrawal. In steady state (between 800 and $1000 \mathrm{~s}$ ), the upstream discharge is $10 \mathrm{l} / \mathrm{s}$ higher than the initial one, which corresponds exactly to the withdrawal.

The multivariable $H_{\infty}$ controller enables to recover the realtime performance of a pure local upstream controller while ensuring that in steady state, the discharge is delivered by the upstream control (as for a distant downstream controller).

In addition, one observes that the linear simulation reproduces rather accurately the dynamic behavior of the closed-loop system. The main discrepancy concerns the downstream actuator $u_{2}$, which possesses a $5 \mathrm{~mm}$ dead band which is not modelled here. This, together with the important measurement noise explains the difference between the experiment and the simulation. The response times are however very close and the controller reacts as expected.
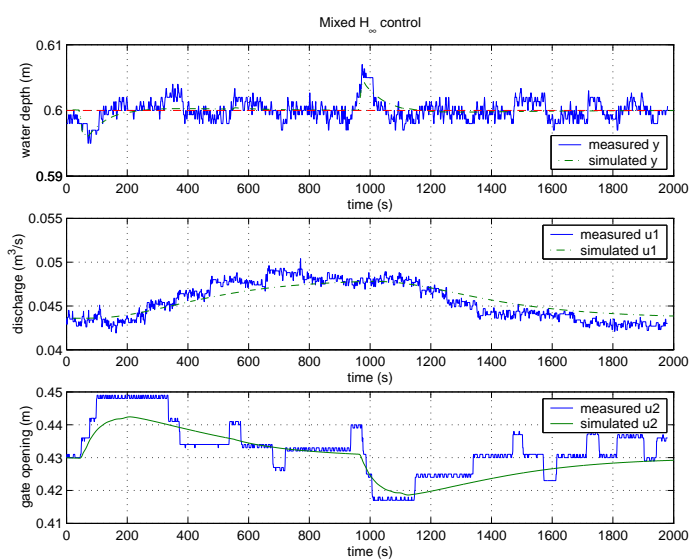

Figure 6: Experimental response of the mixed $H_{\infty}$ controller to a downstream withdrawal, comparison with a linear simulation

\section{Conclusion}

The paper has exposed and validated a methodology to design automatic controllers for irrigation canals, based on the SaintVenant model. The interest of the method is to greatly simplify the model calibration (a single experiment is needed to calibrate the model in steady state). Linear models around different reference points can then be obtained using recent results [5]. The paper has examined the two classical control politics for an irrigation canal pool, namely local upstream and distant downstream control. These two control politics are interpreted as particular monovariable cases of a multivariable control architecture. A mixed control architecture is proposed, that combines the advantages of each control politic, in order to give a solution to the compromise between real-time perfor- mance (obtained with the upstream control politic) and the water resource management (obtained with the downstream control politic). This design problem is formulated as an $H_{\infty}$ optimization problem, using appropriate frequency weighting functions.

It is the first step towards a general methodology for designing controllers realizing a desired compromise between water resource and real-time efficiency. This paper presents the first application (to the best of the authors' knowledge) of $H_{\infty}$ control to an experimental canal.

\section{Acknowledgments}

This work was supported by the french Embassy in Portugal and GRICES (Gabinete de Relações Internacionais da Ciência e do Ensino Superior) of Portugal, through the collaboration project $\mathrm{n}^{\circ}$ 547-B4 : "Modélisation hydraulique et tests de régulateurs automatiques pour le canal réduit d'Évora". We thank Prof. Manuel Rijo from the University of Évora for the opportunity he gave us to use the canal, and for his hospitality during our stay in Portugal.

The authors acknowledge the financial help of Cemagref and INRA through the collaborative program ASS AQUAE $n^{\circ} 02$ on the control of delayed hydraulic systems.

\section{References}

[1] V.T. Chow. Open-channel Hydraulics. McGraw-Hill Book Company, New York, 1988. 680 p.

[2] K. Hoffman. Banach Spaces of Analytic Functions. Prentice Hall, London, 1962.

[3] P.P. Khargonekar and K. Poolla. Robust stabilization of distributed systems. Automatica, 22(1):77-84, 1986.

[4] A. Lencastre. Hydraulique générale. Eyrolles, SAFEGE, 1996.

[5] X. Litrico and V. Fromion. Infinite dimensional modelling of open-channel hydraulic systems for control purposes. In $41^{\text {st }}$ Conf. on Decision and Control, pages 1681-1686, Las Vegas, 2002.

[6] X. Litrico and V. Fromion. Real-time management of multireservoir hydraulic systems using $H_{\infty}$ optimization. In IFAC World Congress, Barcelona, 2002.

[7] X. Litrico, V. Fromion, J.P. Baume, and M. Rijo. Modelling and PI controller design for an irrigation canal. In European Control Conference, Cambridge, UK, 2003.

[8] X. Litrico, V. Fromion, and G. Scorletti. Improved performance for open-channel hydraulic systems using intermediate measurements. In IFAC Workshop on Time-Delay Systems, pages 113118, Santa Fe, 2001.

[9] M.J. Shand. Automatic downstream control systems for irrigation canals. Ph.D. thesis, University of California, Berkeley, 1971.

[10] S. Skogestad and I. Postlethwaite. Multivariable Feedback Control. Analysis and Design. Wiley, 1998. 